\title{
Towards Reproducible User Studies and Behavioral Experiments for Cartography With the stimsrv Framework
}

\author{
Florian Ledermann* and Georg Gartner \\ TU Wien, Research Division Cartography, florian.ledermann@tuwien.ac.at \\ * Corresponding author
}

Keywords: Map Use, User Studies, Reproducibility

\begin{abstract}
:
The continued and renewed need for user studies to explore how users perceive and interact with maps and geographic visualizations has been repeatedly stated by scholars of our field (MacEachren \& Kraak, 2001; Roth, 2015; Roth et al., 2017; White, 2017). On a practical level however, there is currently no technological platform available that fulfills all the unique needs of our field for conducting user studies. Some researchers resort to online survey systems as a platform for user research, with their obvious limitations when it comes to accurate reproduction of cartographic depictions or interactive settings; in controlled lab settings, commercial or open-source software developed for behavioral studies may be used, particularly in conjunction with the use of eye tracking hardware; yet other experiments may be implemented "from scratch" or on top of APIs and code libraries. In all three of these scenarios, some common requirements of our field are not addressed by the respective base technologies. Researchers may thus be forced to make compromises in their experiment design, adjust their research questions to what is afforded by the particular tools selected, or invest considerable time and effort to implement base functionality that is a common requirement but not provided by the particular platform used. Furthermore, the recent debate about the "reproducibility crisis" has led to the demand that other researchers be able to re-run experiments themselves, which is often not the case when closed source software or prototype arrangements with intricate configuration of hard- and software are put to use.
\end{abstract}

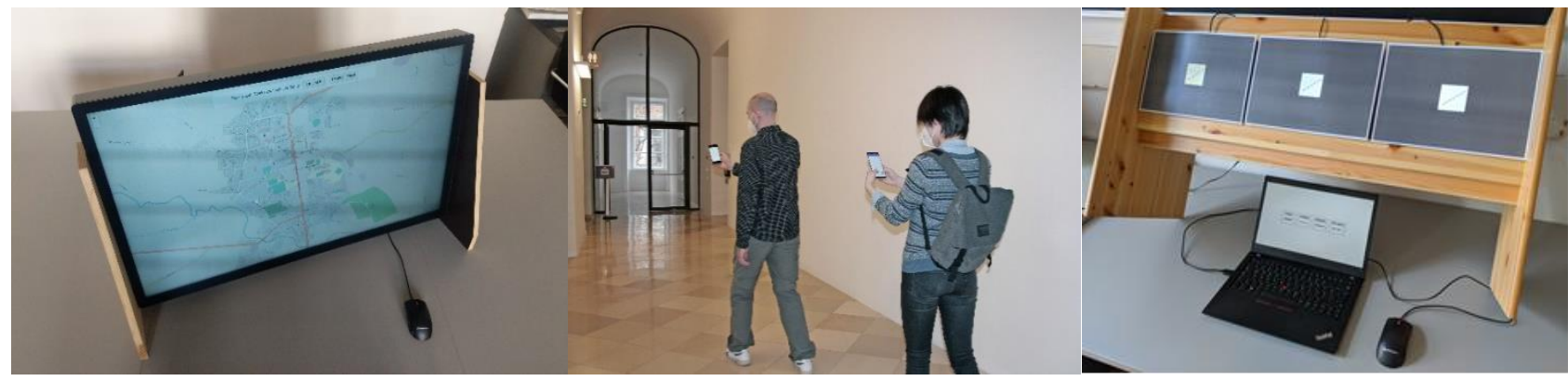

Figure 1. Three case studies implemented with stimsrv, our proposed framework for user studies in cartography and geovisualization. Left: map use / map interaction study; middle: "Wizard of Oz"-style prototyping of indoor navigation interface; right: Comparing cartographic symbols across different displays.

In this paper, we present a framework for implementing user studies and behavioral experiments that has been developed in the context of a cartography research group and therefore specifically addresses some of the unique requirements of our field. In particular we have identified the following functional requirements to support state-of-theart cartographic research: (a) accurate reproduction of visual stimuli across a wide range of devices, media and viewing conditions (b) maps and location tracking as readily available, reusable and highly configurable components to build upon (c) support for heterogeneous distributed setups including mobile devices (d) fast event distribution and timestamp synchronization across devices for real-time interaction and integration of event-stream sensors such as eye trackers or location tracking devices (e) logging of user actions and event streams (such as (map) interaction, location tracking or eye tracking) (f) composition of experiments from existing, modified, extended and custom-made components, such that component libraries for cartographic experiments can be developed within research groups and shared across the cartographic and geosciences community. Furthermore, to aid reproducibility and peer review of experiments, facilitate scientific collaboration and allow for use in teaching and student's research, the following nonfunctional requirements have been identified: $(\mathrm{g})$ a simple conceptual model tailored towards behavioral experiments and user studies (h) a centralized experiment specification that defines the behavior of all (potentially distributed) components (i) support of multiple configuration scenarios to aid development, simulation runs and different experiment configurations $(\mathrm{j})$ publication under an open source license. 
Our framework, stimsrv, strives to meet the requirements listed above and is therefore, to our knowledge, unique in the realm of potential technological foundations available for user studies and behavioral experiments. stimsrv is implemented in JavaScript and uses modern web browsers as its primary "frontend" technology. However, in order to fulfill the requirements of distributed setups and the support of a wide range of devices, parts of the JavaScript code can be run on the server in order to coordinate multiple clients in a distributed setup, and the user interface can be rendered on the server and sent to the client as an image, in order to support devices with simple or outdated web browsers such as e-book readers or older mobile phones. This allows the inclusion of a wide range of devices for stimulus output and response input, while keeping a single codebase written in modern JavaScript even for devices which do not support it.

The proposed framework has been described elsewhere in detail (Ledermann \& Gartner, 2021). In this paper we focus on presenting aspects of the framework specific to cartographic studies, and present a series of case studies related to our field. The first case study implements a classic map use scenario, asking participants to locate places specified only by their name on an interactive map, tracking their zooming and panning behavior across different interaction modalities. The second case study employs "Wizard of Oz"-style prototyping (Kelley, 1984) in a mobile indoor navigation scenario, in which the participant is presented a seemingly interactive navigation interface which is in fact controlled manually by the experimenter. The third case study compares the rendering of cartographic symbols on displays of varying pixel density, using multiple different mobile phones for stimulus presentation and another mobile device for participant response (Ledermann, 2021).

In each of the case studies, features which can be complex to implement but are supported by the stimsrv framework such as distribution across multiple devices, event synchronization, logging of participant responses, or conversion of real-world (metric) dimensions to screen pixels, could be used effortlessly without additional implementation overhead by the study designer. The first study had originally been implemented in plain JavaScript without the help of a framework or API other than the Leaflet mapping API, which allows a comparison of the code and its complexity to the re-implementation using stimsrv. In the new implementation, additional features such as a supervisor screen mirroring the participant's actions and fine-grained event logging were realized, while at the same time reducing the number of lines of code necessary to only $25 \%$ of the original code. Complex code constructs such as functions, loops and conditionals could also be reduced to $25 \%$ or less of the original, and in some instances removed completely. While this is only an initial, informal report and a more thorough evaluation of stimsrv is still to be undertaken, it shows the potential for reducing code complexity while at the same time realizing additional important functional and nonfunctional requirements of cartographic user studies, therefore (i) allowing non-expert programmers to implement more complex and/or complete experiments as part of their (thesis) work, (ii) allowing experts to accomplish multiple and/or more complex experiments with equal investment of time and resources and (iii) supporting peers in reviewing and reproducing experiments due to better code legibility, simpler and less code, the re-use of common building blocks of our field and the centralized definition in one place of even complex distributed setups.

A future goal in the context of teaching is to allow students without prior programming experience to implement their own distributed user studies with stimsrv after a $5 \times 2$ hours introduction to JavaScript and a brief introduction to the framework - a challenge that we consider impossible to fulfil with conventional web mapping technologies within our given teaching framework according to our experience. This would create the opportunity to more thoroughly evaluate the proposed framework in real-world research scenarios.

\section{Bibliography}

Kelley, J. F. (1984). An iterative design methodology for user-friendly natural language office information applications. ACM Transactions on Information Systems, 2(1), 26-41. https://doi.org/10.1145/357417.357420

Ledermann, F. (2021). Investigating the Effect of Display Pixel Density on the Minimal Legible Size of Cartographic Symbols. Abstracts of the 30th International Cartographic Conference (ICC 2021).

Ledermann, F., \& Gartner, G. (2021). Towards Conducting Reproducible Distributed Experiments in the Geosciences. AGILE: GIScience Series, 2, 1-7. https://doi.org/10.5194/agile-giss-2-33-2021

MacEachren, A. M., \& Kraak, M.-J. (2001). Research challenges in geovisualization. Cartography and Geographic Information Science, 28(1), 3-12.

Roth, R. E. (2015). Challenges for Human Subjects Research in Cartography. Proceedings of the ICA Workshop on Envisioning the Future of Cartographic Research, Curitiba, Brazil, 21.

Roth, R. E., Çöltekin, A., Delazari, L., Filho, H. F., Griffin, A., Hall, A., Korpi, J., Lokka, I., Mendonça, A., \& Ooms, K. (2017). User studies in cartography: Opportunities for empirical research on interactive maps and visualizations. International Journal of Cartography, 3(sup1), 61-89.

White, T. M. (2017). From Existing Practices to Best Practices: Improving the Quality and Consistency of Participant Assessment Methods in Cartographic User Studies [PhD Thesis]. University of Kansas. 